\title{
Analyst
}

Cite this: Analyst, 2014, 139, 3314

\section{CotA laccase: high-throughput manipulation and analysis of recombinant enzyme libraries expressed in $E$. coli using droplet-based microfluidics $\uparrow$}

\author{
Thomas Beneyton, ${ }^{\mathrm{ab}}$ Faith Coldren, ${ }^{\mathrm{a}}$ Jean-Christophe Baret, ${ }^{\mathrm{cd}}$ Andrew D. Griffiths ${ }^{\mathrm{ab}}$ \\ and Valérie Taly*aé
}

We present a high-throughput droplet-based microfluidic analysis/screening platform for directed evolution of CotA laccase: droplet-based microfluidic modules were combined to develop an efficient system that allows cell detection and sorting based on the enzymatic activity. This platform was run on two different operating modes: the "analysis" mode allowing the analysis of the enzymatic activity in droplets at very high rates $(>1000 \mathrm{~Hz})$ and the "screening" mode allowing sorting of active droplets at $400 \mathrm{~Hz}$. The screening mode was validated for the directed evolution of the cytoplasmic CotA laccase from $B$. subtilis, a potential interesting thermophilic cathodic catalyst for biofuel cells. Single $E$. coli cells expressing either the active $\operatorname{Cot} A$ laccase $(E$. coli $\operatorname{Cot} A)$ or an inactive frameshifted variant $(E$. $\operatorname{coli} \Delta \operatorname{Cot} A)$ were compartmentalized in aqueous droplets containing expression medium. After cell growth and protein expression within the droplets, a fluorogenic substrate was "picoinjected" in each droplet. Fluorescence-activated droplet sorting was then used to sort the droplets containing the desired activity and the corresponding cells were then recultivated and identified using colorimetric assays. We demonstrated that $E$. coli $\operatorname{Cot} A$ cells were enriched 191-fold from a $1: 9$ initial ratio of $E$. coli $\operatorname{Cot} A$ to E. coli $\triangle$ CotA cells (or 437 -fold from a $1: 99$ initial ratio) using a sorting rate of 400 droplets per s. This system allows screening of $10^{6}$ cells in only $4 \mathrm{~h}$, compared to 11 days for screening using microtitre

Received 30th January 2014 Accepted 11th March 2014

DOI: 10.1039/c4an00228h

www.rsc.org/analyst plate-based systems. Besides this low error rate sorting mode, the system can also be used at higher throughputs in "enrichment" screening mode to make an initial purification of a library before further steps of selection. Analysis mode, without sorting, was used to rapidly quantify the activity of a $\operatorname{CotA}$ library constructed using error-prone PCR. This mode allows analysis of $10^{6}$ cells in only $1.5 \mathrm{~h}$.

\section{Introduction}

Directed evolution is a powerful biotechnological tool that mimics and accelerates natural evolution at the laboratory scale. It relies on inducing artificial mutations to create populations of new variants and selecting the rare ones which exhibit improved functionality under a user-defined and

\footnotetext{
${ }^{a}$ Laboratoire de Biologie Chimique, Institut de Science et d'Ingénierie Supramoléculaires (ISIS), CNRS UMR 7006, 8 allée Gaspard Monge, F-67083 Strasbourg, France

${ }^{b}$ Laboratoire de Biochimie, Ecole Supérieure de Physique et de Chimie Industrielles de la ville de Paris (ESPCI ParisTech), CNRS UMR 7084, 10 rue Vauquelin, F-75231, Paris, France

'Droplets Membranes and Interfaces, Max Planck Institute for Dynamics and SelfOrganization, Am Fassberg, 17, D-37077 Goettingen, Germany

${ }^{d}$ Université de Bordeaux, CRPP-CNRS, 115 Avenue Schweitzer, F-33600 Pessac, France 'Université Paris Sorbonne Cité, INSERM UMR-S1147, 45 rue des Saints-Pères, F75270, Paris, France. E-mail: valerie.taly@parisdescartes.fr

$\dagger$ Electronic supplementary information (ESI) available: One picoinjection movie, scheme of the LabView program and a detailed description of microfluidic modules. See DOI: 10.1039/c4an00228h
}

controlled selection pressure. It has been intensively used this last decade to improve enzymes ${ }^{1}$ or microorganism strains $s^{2}$ for industrial applications or, for example, to study the fundamentals of evolution itself. ${ }^{3}$ The impact of directed evolution is however limited by its need for efficient high-throughput screening methods, especially when selection occurs on the enzymatic activity. Indeed, efficient directed evolution requires screening of very large libraries under conditions that closely match the desired activity. As genetic information is encoded in the genotype and the selection is based on phenotypic activities, genotype and phenotype have to be linked. Ideally, they must be physically linked by compartmentalization as it is in cells. Nowadays, the most controlled and flexible high-throughput enzymatic activity screening technique is the robotized compartmentalization of assays in microtitre plates, allowing us to process up to 1 assay per second. This technology has even reached its physical limits as reducing assay volumes to below $1 \mu \mathrm{L}$ is problematic due to evaporation and capillarity. ${ }^{4}$ One way to miniaturize reaction vessels exploits microarrays and microfluidic lab-on-chip technology, decreasing the reaction volume to $\sim 250 \mathrm{pL}^{5}{ }^{5}$ Nevertheless, these screening techniques 
are still limiting the applicability of directed evolution. ${ }^{6}$ Another way is to use in vitro compartmentalization (IVC): genotype and phenotype are linked by compartmentalizing individual components of a library in water-in-oil droplets. ${ }^{7}$ Initially used with bulk emulsion, IVC is suitable for in vitro evolution, reducing the reaction volume below $1 \mathrm{pL}$ and allowing screening of large libraries (up to $10^{11}$ ). ${ }^{8}$ However, the range of activities that can be screened and the flexibility of the method are rather limited because of the lack of control of the droplet volume and timing. Moreover, most assays require the addition of new reagents at defined times to initiate or terminate a reaction. As bulk emulsions are hardly manipulated, the applicability of IVC is markedly limited in that case. These limitations can be overcome by using the droplet-based microfluidic technology, which offers the possibility to use IVC in a highly controlled manner.

Indeed, droplet-based microfluidics allows the production of highly monodisperse water-in-oil droplets and the manipulation of those at a single level and at very high frequencies. ${ }^{9}$ During recent years, this technology has been subjected to increasing interest for screening applications, which has led to intensive developments of efficient tools to handle single droplets within an emulsion. Different droplet-based microfluidic modules have been developed to perform controlled droplet manipulation, such as fusion, ${ }^{\mathbf{1 0 - 1 2}}$ picoinjection, ${ }^{\mathbf{1 3 , 1 4}}$ incubation ${ }^{15,16}$ or sorting based on fluorescence, ${ }^{17}$ especially in the droplets' volume range of $10 \mathrm{pL}$ to $100 \mathrm{pL}$, suitable for biological assay and cell handling. This technology has arrived at the point where single modules can be combined to create efficient integrated screening platforms. ${ }^{18}$ However, very few directed evolution platforms have been described in the literature. An integrated encapsulation/fusion/sorting platform has been developed for complete in vitro directed evolution. ${ }^{19}$ In vivo screening platforms have also been described but their flexibility is limited as they only consist of encapsulation/sorting techniques. ${ }^{17,20-22}$ A flexible and efficient in vivo screening platform for directed evolution would allow the growth of cells within droplets and then the addition of the substrate to initiate the enzymatic reaction at a controlled time prior to sorting.

CotA laccase is an oxidoreductase (EC 1.10.3.2) that catalyzes the one-electron oxidation of phenols, polyphenols and anilines with the concomitant four-electron reduction of $\mathrm{O}_{2}$ to water. ${ }^{23}$ Due to their broad substrate specificity and few requirements, laccases such as CotA have high interest for green industrial applications, such as delignification of wood, biosensors or biofuel cells. ${ }^{24}$ We designed a flexible droplet-based microfluidic platform that can be used for high-throughput screening of E. coli cells for the CotA enzymatic activity (Fig. 1). This platform involves the following steps: (i) encapsulation of cells in aqueous droplets; (ii) incubation for cell growth and protein expression in droplets; (iii) picoinjection of the fluorogenic substrate; (iv) incubation for enzymatic reactions; (v) sorting of droplets based on the enzymatic activity according to the fluorescence intensity; (vi) recovery of cells from the sorted droplets. We demonstrated that this high-throughput screening platform allows enrichment of specific cells according to the CotA enzymatic activity and should be a powerful tool to screen large

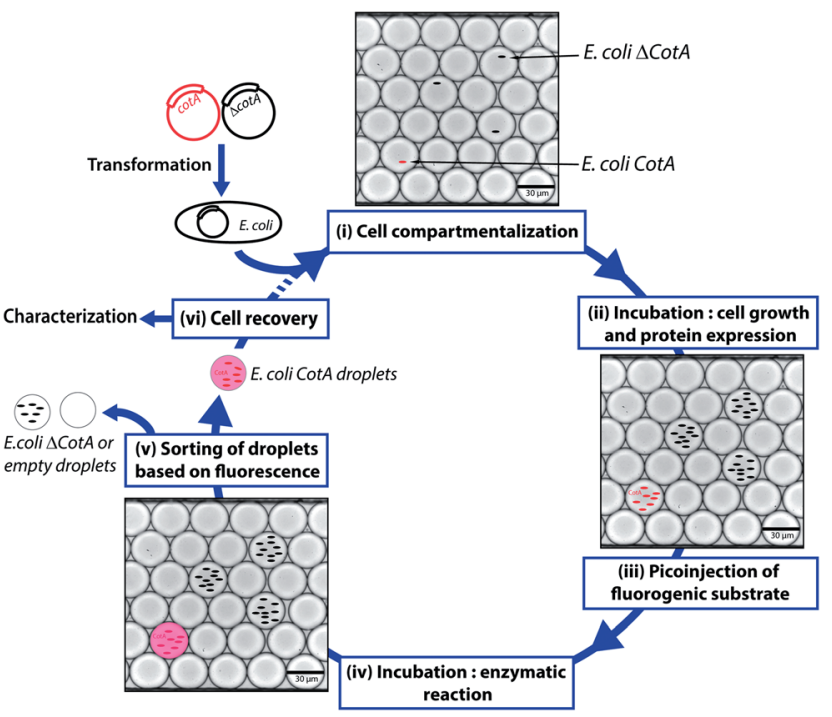

Fig. 1 Schematic of the droplet-based microfluidic model screening platform. Wild type CotA gene and a frameshifted inactive variant $\triangle$ CotA gene were transformed into $E$. coli cells to give $E$. coli $\operatorname{Cot} A$ and $E$. coli $\triangle \operatorname{Cot} A$ strains. (i) A mixture of $E$. coli $\operatorname{Cot} A / E$. coli $\triangle \operatorname{Cot} A$ cell suspensions was encapsulated in droplets containing induction medium. (ii) The emulsion was incubated off-chip for cell growth and CotA or $\Delta$ CotA enzyme expression within the droplets. (iii) Fluorogenic substrate was picoinjected in every droplet. (iv) The emulsion was incubated off-chip for the enzymatic reaction. (v) The droplets were sorted based on the enzymatic activity according to the fluorescence intensity. (vi) Cells were recovered from the sorted droplets and could be either characterized to determine enrichment in E. coli CotA cells or engaged in another round of selection.

libraries for CotA directed evolution experiments. Furthermore, the droplet-based microfluidic platform provides also a powerful high-throughput cell analysis tool to quickly quantify the distribution of the enzymatic activity within a library. We created a library of CotA variants by error-prone PCR and analyzed the library of cells obtained by transformation of the library. In general, such screening tools could be extended to many other laccases or oxidoreductases, enzymes of high industrial interest.

\section{Experimental}

\subsection{Reagents}

2,2'-Azino-bis(3-ethylbenzothiazoline-6-sulfonic acid) (ABTS), ampicillin, D-glucose, ethylenediaminetetraacetic acid (EDTA), and copper(II) chloride were purchased from Sigma-Aldrich. Isopropyl $\beta$-D-1-thiogalactopyranoside (IPTG), LB medium and LB agar were obtained from Roth. Dextran Texas Red (10 $000 \mathrm{MW}$ ), dextran fluorescein (10 $000 \mathrm{MW}$ ) and fluorescein were purchased from Life Technologies.

\subsection{Fabrication of microfluidic devices}

Microfluidic devices were fabricated from poly(dimethylsiloxane) (PDMS) using standard soft-lithography methods. ${ }^{25}$ A mold of SU8-2015 negative photoresist (MicroChem Corp.) was prepared on a silicon wafer (Siltronix) by UV exposure 
(MJB3 contact mask aligner; SUSS MicroTec) through a photolithography mask (Selba SA) and subsequent development (SU-8 developer; MicroChem Corp.). A curing agent was added to the PDMS base (Sylgard 184 elastomer kit; Dow Corning Corp.) to a final concentration of $10 \%(\mathrm{w} / \mathrm{w})$, mixed, and poured onto the mold. After degassing under vaccum to remove air bubbles, the mold was incubated for several hours at $65{ }^{\circ} \mathrm{C}$ for PDMS crosslinking. PDMS was then peeled off and inputs and outputs were punched with a $0.75 \mathrm{~mm}$ diameter biopsy punch. The structured side of the PDMS slab was bound to a $75 \times 50 \times 1 \mathrm{~mm}$ glass microscope slide (Corning) by exposing both parts to an oxygen plasma (Plasma system FEMTO; Diener Electronic). The device was incubated for several minutes at $65{ }^{\circ} \mathrm{C}$. Finally, an additional hydrophobic surface coating was applied to the microfluidic channel walls by injecting the channels with $1 \%$ (w/w) $1 H, 1 H, 2 H, 2 H$-perfluorodecyltrichlorosilane (97\%; ABCR) in HFE7100 fluorinated oil (3 M).

Electrodes were included in the microfluidic device as additional channels which were filled with metal: the device was heated at $110{ }^{\circ} \mathrm{C}$ and a $51 \mathrm{In} / 32.5 \mathrm{Bi} / 16.5 \mathrm{Sn}$ low-temperature solder (Indium Corp.) was melted inside the electrode channels. ${ }^{26}$ Electrical connections with the solder electrodes were made with short pieces of electrical wire (Radiospares) and strengthened with Loctite 3526 UV glue (Epotecny).

\subsection{Optical setup, data acquisition and control system}

The optical setup consisted of an Axiovert 200 inverted microscope (Carl Zeiss SAS) mounted on a vibration-dampening platform (Thorlabs GmbH). A $20 \mathrm{~mW}, 488 \mathrm{~nm}$ solid-state laser (LAS; Newporte-Spectraphysics) and a $50 \mathrm{~mW}, 532 \mathrm{~nm}$ solidstate laser (CrystaLaser) were focused through the objective lens $(40 \times / 0.6$; Carl Zeiss SAS $)$ across a channel within the microfluidic device. Light emitted from fluorescing droplets was captured by the objective, channeled back along the path of the lasers. The emitted light was separated from the laser beam and split between two photomultiplier tubes (PMT, H5784-20; Hamamatsu), which captured the light through a $510 \mathrm{~nm}$ bandpass filter (510/20-25; Semrock Inc.) for the green PMT and through a $617 \mathrm{~nm}$ bandpass filter (617/73-25, Semrock Inc.) for the orange PMT. The signal output from the PMT was analyzed using a PCI-7831R Multifunction Intelligent Data Acquisition (DAQ) card (National Instruments Corporation) executing a program written in LabView 8.2 (FPGA module, National Instruments Corporation, Fig. S1, ESI $\dagger$ ), which allowed the identification of droplets by peaks in fluorescence, as well as the width of each droplet. The data acquisition rate for the system was $100 \mathrm{kHz}$. A Phantom v4.2 high speed digital camera (Vision Research) was mounted on the top camera port of the microscope to capture digital images during droplet manipulation.

Liquids were pumped into the microfluidic devices using standard-pressure infusion-only PHD 22/2000 syringe pumps (Harvard Apparatus Inc.). Syringes (Omnifix-F®; BBRAUN) were connected to the microfluidic devices using $0.6 \times 25 \mathrm{~mm}$ needle (Terumo) and PTFE tubing with an internal diameter of $0.56 \mathrm{~mm}$ and an external diameter of $1.07 \mathrm{~mm}$ (Fisher Scientific).

\subsection{Description of microfluidic devices}

We used aqueous droplets in HFE7500 fluorinated oil (3 M) stabilized against coalescence by block-polymer homemade surfactant, named KryJeffa900 in this report. KryJeffa900 surfactant was prepared from the commercially available carboxylic acid Krytox157-FSH (Dupont) and Jeffamine ${ }^{\circledR}$ polyetheramines (ED 900, Huntsmann) following a synthesis route based on the one described by Holtze et al. ${ }^{27}$

All the microfluidic devices were fabricated with a channel depth of $25 \mu \mathrm{m}$. Four different modules were used to compose the platform.

First, a dropmaker module (Fig. S2, ESI $\dagger$ ) was used to generate $14 \mathrm{pL}$ droplets (coefficient of variance $(\mathrm{CV})=3 \%$ ) at $1600 \mathrm{~Hz}$ by flow-focusing of the aqueous stream $\left(80 \mu \mathrm{L} \mathrm{h}^{-1}\right)$ with two streams of HFE7500 fluorinated oil (3 M) $\left(190 \mu \mathrm{L} \mathrm{h}^{-1}\right)$ containing $1 \%(\mathrm{w} / \mathrm{w})$ of KryJeffa900 surfactant. A dual-dropmaker module (Fig. S3, ESI $\dagger$ ) was used to generate two $14 \mathrm{pL}$ droplets of emulsions simultaneously (the same conditions of operation). The generated emulsions flowed off-chip through PTEF tubing to a syringe.

The second module was a picoinjection device (Fig. S4 and ESI movie "picoinjection.avi", ESI $\dagger$ ) in which droplets were reloaded (70-160 $\mu \mathrm{L} \mathrm{h}^{-1}$ ) and spaced-out at a flow-focusing junction (Fig. 3b) with HFE7500 fluorinated oil (3 M) (200$600 \mu \mathrm{L} \mathrm{h}^{-1}$ ) containing $1 \%(\mathrm{w} / \mathrm{w})$ of KryJeffa900 surfactant. The picoinjected phase was loaded into a $250 \mu \mathrm{L}$ gas-tight syringe (Hamilton Company) and pumped into the device (Fig. 3b). A continuous AC field $(20 \mathrm{kHz} ; 200 \mathrm{~V})$ was applied across the electrodes and more than $99.5 \%$ of the droplets were picoinjected ( $\sim 1 \mathrm{pL}$; estimated by video analysis) at $1500-3000 \mathrm{~Hz}$ (working range of the module) to give droplets of $\sim 15 \mathrm{pL}(\mathrm{CV}=$ 4.5\%). The generated emulsions flowed off-chip through PTEF tubing to a glass vial immerged in ice to slow down the enzymatic reaction. The off-chip incubation time at room temperature $\left(25^{\circ} \mathrm{C}\right)$ was determined by the length of the PTFE tubing connected from the chip to the glass vial: droplets were incubated at $25{ }^{\circ} \mathrm{C}$ while they were flowing in to the vial (collection) and from the vial (reinjection).

Under screening mode operation, the last module was fluorescence-activated droplet sorting (FADS) ${ }^{16}$ (Fig. S5, ESI $\dagger$ ) in which droplets were reloaded $\left(20-70 \mu \mathrm{L} \mathrm{h}^{-1}\right)$ and spaced-out at a flow-focusing junction (Fig. 3c) with HFE7500 fluorinated oil (3 M) (500-1000 $\left.\mu \mathrm{L} \mathrm{h}^{-1}\right)$. The droplets were analyzed by the optical setup and fluorescent droplets were sorted at $400 \mathrm{~Hz}$ by applying an AC field pulse (30 kHz; 1000-1400 V; $0.5 \mathrm{~ms}$ ). The sorted droplets were collected in a $1.5 \mathrm{~mL}$ microcentrifuge tube (Axygen Inc.).

Under analysis mode operation, the last module was a reloading module (Fig. S6, ESI $\dagger$ ) in which droplets were reloaded (50-150 $\mu \mathrm{L} \mathrm{h}^{-1}$ ) and spaced-out at a flow-focusing junction with HFE7500 fluorinated oil (3 M) (200-400 $\left.\mu \mathrm{L} \mathrm{h}^{-1}\right)$. The droplets were detected by the optical setup and analyzed at $\sim 1000 \mathrm{~Hz}$.

\subsection{Plasmid construction}

The plasmid pLOM10 coding for the wild type CotA laccase was kindly provided by Prof. L. O. Martins (Universidade Nova de 
Lisboa). The plasmid pLOMP3 coding for the inactive frameshifted variant $\Delta$ CotA was constructed starting from pLOM10. A mixture of $44 \mu \mathrm{L}$ of pLOM10 (35 ng $\left.\mu \mathrm{L}^{-1}\right), 5 \mu \mathrm{L}$ of NEB4 buffer $10 \times$ (New England Biolabs) and $1 \mu \mathrm{L}$ of AatII enzyme $\left(20 \mathrm{U}^{-1}\right.$; New England Biolabs) was incubated for $15 \mathrm{~h}$ at $37^{\circ} \mathrm{C}$. T4 DNA Polymerase (3U; New England Biolabs) and $200 \mu \mathrm{M}$ of dNTP (New England Biolabs) were added and the mixture was incubated for $15 \mathrm{~min}$ at $12{ }^{\circ} \mathrm{C}$. The reaction was quenched by adding $10 \mathrm{mM}$ of EDTA and the mixture was incubated for $20 \mathrm{~min}$ at $75{ }^{\circ} \mathrm{C}$. After purification using the PCR purification kit (Qiagen), $10 \mu \mathrm{L}$ of DNA (18 ng $\mu \mathrm{L}^{-1}$ ) was mixed with $2 \mu \mathrm{L}$ of T4 DNA ligase buffer $10 \times$ (New England Biolabs), $1 \mu \mathrm{L}$ of T4 DNA ligase (400 U $\mu \mathrm{L}^{-1}$; New England Biolabs) and $7 \mu \mathrm{L}$ of ultrapure water. The mixture was incubated for $3 \mathrm{~h}$ at $25^{\circ} \mathrm{C}$. The plasmid was then transformed into Xl10 gold $E$. coli competent cells (Stratagene). 12 colonies were isolated and sequenced.

\subsection{Preparation of cell suspensions for encapsulation}

Plasmids pLOM10 and pLOMP3 were transformed into E. coli C41(DE3) ${ }^{28}$ using standard molecular biology protocols to give an active strain (E. coli CotA) and an inactive strain (E. coli $\triangle$ CotA) in which the CotA or $\Delta$ CotA protein could be produced under the control of the T7lac promoter. $2 \mathrm{~mL}$ of LB medium containing ampicillin $\left(0.1 \mathrm{mg} \mathrm{mL}^{-1}\right)$ was inoculated with a single colony of the desired strain. This aliquot was grown for $14 \mathrm{~h}$ at $37^{\circ} \mathrm{C}(230$ $\mathrm{rpm})$. The following day, $50 \mathrm{~mL}$ of fresh $\mathrm{LB}$ medium containing ampicillin $\left(0.1 \mathrm{mg} \mathrm{mL}^{-1}\right)$ and $\mathrm{CuCl}_{2}(0.25 \mathrm{mM})$ was inoculated with $500 \mu \mathrm{L}$ of preculture. The culture was grown under the same conditions to reach an $\mathrm{OD}_{600}$ of $0.6 .5 \mathrm{~mL}$ of culture was centrifuged ( $10 \mathrm{~min} ; 2000 \mathrm{~g} ; 4^{\circ} \mathrm{C}$ ) and the pellet was resuspended in $5 \mathrm{~mL}$ of fresh $\mathrm{LB}$ medium containing ampicillin $(0.1 \mathrm{mg}$ $\left.\mathrm{mL}^{-1}\right), \mathrm{CuCl}_{2}(0.25 \mathrm{mM})$ and IPTG $(1 \mathrm{mM})$ for induction of protein expression. $\mathrm{OD}_{600}$ was measured and the culture was diluted to have the desired number of cells per droplet volume ( $\mathrm{OD}_{600}$ of 1 corresponded to $5 \times 10^{8}$ cells per $\mathrm{mL}$ ).

\subsection{CotA and $\Delta$ CotA enzymatic activity in droplets}

The dual-dropmaker module (Fig. S2, ESI $\dagger$ ) was used to produce two emulsions simultaneously. E. coli CotA cell suspension was encapsulated in $14 \mathrm{pL}$ droplets containing $1 \mu \mathrm{M}$ of dextran fluorescein while $E$. coli $\triangle$ CotA cell suspension was encapsulated in $14 \mathrm{pL}$ droplets containing $5 \mu \mathrm{M}$ of dextran fluorescein. The binary emulsion was incubated for $15 \mathrm{~h}$ at $30{ }^{\circ} \mathrm{C}$ and reloaded into the picoinjection module (Fig. S3, ESI $\dagger$ ). LB medium containing Amplex®UltraRed (0.5 $\mu \mathrm{M}$; Life Technologies) was used as the picoinjected phase. After $10 \mathrm{~min}$ of incubation at room temperature $\left(25{ }^{\circ} \mathrm{C}\right)$, the emulsion was then reloaded into the reloading module (Fig. S5, ESI $\dagger$ ) for analysis.

\subsection{Screening mode: model selection}

E. coli CotA and E. coli $\triangle$ CotA cell suspensions were mixed together in the desired ratio prior to encapsulation using the dropmaker module (Fig. S1, ESI $\dagger$ ). The emulsion was incubated for $15 \mathrm{~h}$ at $30{ }^{\circ} \mathrm{C}$ and reloaded into the picoinjection module (Fig. S3, ESI $\dagger$ ). LB medium containing Amplex®UltraRed (0.5 $\mu \mathrm{M}$; Life Technologies) was used as the picoinjected phase.
After $10 \mathrm{~min}$ of incubation at room temperature $\left(25^{\circ} \mathrm{C}\right)$, the emulsion was then reloaded into the FADS module (Fig. S4, ESI $\dagger$ ) for sorting. The sorted droplets were recovered by cutting the collection PTFE tubing and draining last droplets into the collection microcentrifuge tube. To dislodge any droplets remaining in the tubing, it was flushed with $200 \mu \mathrm{L}$ of fresh $\mathrm{LB}$ medium. All the contents of the collection microcentrifuge tube were added to $10 \mathrm{~mL}$ of fresh LB medium containing ampicillin $\left(0.1 \mathrm{mg} \mathrm{mL} \mathrm{m}^{-1}\right)$ and grown for $15 \mathrm{~h}$ at $37{ }^{\circ} \mathrm{C}$. As a reference, $250 \mu \mathrm{L}$ of the initial emulsion was grown under the same conditions.

Cells before and after sorting were then identified using two different techniques. On one hand, the sorted cells were plated on LB agar containing ampicillin (0.1 $\left.\mathrm{mg} \mathrm{mL}{ }^{-1}\right), \mathrm{CuCl}_{2}$ (0.25 mM), IPTG (1 mM) and ABTS ( $5 \mathrm{mM})$, and incubated for 15 $\mathrm{h}$ at $30^{\circ} \mathrm{C}$. Plates were then kept at $4{ }^{\circ} \mathrm{C}$ for $48 \mathrm{~h}$ for coloration to occur. E. coli CotA colonies appeared dark brown while E. coli $\triangle$ CotA colonies remained colourless (Fig. $3 \mathrm{~d}_{2}$ ).

On the other hand, sorted cells were plated on LB agar containing ampicillin $\left(0.1 \mathrm{mg} \mathrm{mL}^{-1}\right)$ and incubated for $15 \mathrm{~h}$ at 37 ${ }^{\circ} \mathrm{C} .87$ colonies were transplanted in a 96-deepwells microtitre plate (Nunc) in $1 \mathrm{~mL}$ of fresh LB medium containing ampicillin (0.1 $\left.\mathrm{mg} \mathrm{mL}^{-1}\right), \mathrm{CuCl}_{2}(0.25 \mathrm{mM})$ and D-glucose $(20 \mathrm{mM})$ with 3 positive controls (E. coli CotA), 3 negative controls (E. coli $\triangle$ CotA) and 3 blanks (growth medium). The microtitre plate was covered by a gas-permeable membrane (Thermofisher) and incubated for $15 \mathrm{~h}$ at $37{ }^{\circ} \mathrm{C}(250 \mathrm{rpm})$. The microtitre plate was then centrifuged (10 min; $1000 \mathrm{~g}$ ) and the medium was replaced with fresh LB medium containing ampicillin $\left(0.1 \mathrm{mg} \mathrm{mL}{ }^{-1}\right), \mathrm{CuCl}_{2}$ $(0.25 \mathrm{mM})$, and IPTG $(1 \mathrm{mM})$. The microtitre plate was covered by a gas-permeable membrane (Thermofisher) and incubated for $15 \mathrm{~h}$ at $30{ }^{\circ} \mathrm{C}(250 \mathrm{rpm})$. The CotA enzymatic activity corresponding to each well was tested in a standard 96-well microtitre plate by mixing $50 \mu \mathrm{L}$ of cells with $50 \mu \mathrm{L}$ of acetate buffer (50 $\mathrm{mM}$; pH 4.5) containing ABTS (5 mM). The CotA enzymatic activity resulted in green coloration of the solution (Fig. $3 \mathrm{~d}_{1}$ ).

\subsection{Library construction}

Primers CotAprom (5'-TGTGACTCAGATCTCGATCCCGCGAA ATTAATAC- $3^{\prime}$ ) and CotAterm (5'-GGTTATGCTAGTTATTGCT CAGCGG-3' ${ }^{\prime}$, biotinylated at $5^{\prime}$ ends, were used to PCR-amplify a 1808-bp fragment containing the CotA gene under mutagenesis conditions using the GeneMorph ${ }^{\circledR}$ II Random mutagenesis kit (Stratagene). A mixture of $1 \mu \mathrm{L}$ of plasmid pLOM10 (100 ng $\left.\mu \mathrm{L}^{-1}\right), 2.5 \mu \mathrm{L}$ of CotAprom $(10 \mu \mathrm{M}), 2.5 \mu \mathrm{L}$ of CotAterm $(10 \mu \mathrm{M}), 1 \mu \mathrm{L}$ of dNTPs ( $5 \mathrm{mM}$ each, New England Biolabs), $1 \mu \mathrm{L}$ of Mutazyme® II $\left(2.5 \mathrm{U}_{\mu} \mathrm{L}^{-1}\right), 5 \mu \mathrm{L}$ of buffer $(10 \times)$ and $37.5 \mu \mathrm{L}$ of RNase/DNase free water (Roth) was subjected to the PCR reaction in a MJ Research thermocycler. The thermal cycle included $240 \mathrm{~s}$ initial denaturation at $94{ }^{\circ} \mathrm{C}$ followed by 30 cycles of thermal cycling and $420 \mathrm{~s}$ of final extension at $72{ }^{\circ} \mathrm{C}$. The thermal cycling protocol consisted of $30 \mathrm{~s}$ at $94{ }^{\circ} \mathrm{C}, 30 \mathrm{~s}$ at $55^{\circ} \mathrm{C}$, and $120 \mathrm{~s}$ at $72{ }^{\circ} \mathrm{C}$. The PCR products were captured using streptavidin coated magnetic beads (Dynabeads® M-280, kilobaseBinder ${ }^{\mathrm{TM}}$ kit, Invitrogen) and released from the magnetic beads using the two cloning sites, BglII and NotI. 
An "easy-purification" acceptor vector pET-TB was constructed by modifying pET-21a $(+)$ vector (Novagen): pET-21a $(+)$ was digested at BglII/NotI cloning sites and a small oligonucleotide cassette was inserted using $\mathrm{K}_{1}$ ( $5^{\prime}$-GGCCGCAAGCTTCA$3^{\prime}$ ) and $\mathrm{K}_{2}$ (5'-GATCTGAAGCTTGC-3') oligonucleotides phosphorylated at the $5^{\prime}$ end. This plasmid pET-TB was easily purified prior to the cloning step as its digestion at BglII and NotI cloning sites generates a small 14-bp fragment, easily eliminated using a PCR purification kit (Qiagen).

The purified mutagenesis PCR product was inserted into the acceptor vector pET-TB between BglII and NotI cloning sites to give the library plasmid pLOM10mut.

\subsection{Analysis mode: library analysis}

The library cell suspension was produced as follows: $4 \mu \mathrm{L}$ of plasmid pLOM10mut was transformed by electroporation into $480 \mu \mathrm{L}$ of $E$. coli $\mathrm{C} 41$ (DE3) competent cells $\left(\sim 2 \times 10^{6}\right.$ cells per $\mu \mathrm{g}$ of DNA) using standard molecular biology protocols. Cells were then grown for $15 \mathrm{~h}$ at $37{ }^{\circ} \mathrm{C}(230 \mathrm{rpm})$ in $500 \mathrm{~mL}$ of fresh $\mathrm{LB}$ medium containing ampicillin $\left(0.1 \mathrm{mg} \mathrm{mL}^{-1}\right)$ and D-glucose $(20$ $\mathrm{mM}) .500 \mathrm{~mL}$ of fresh LB medium containing ampicillin $(0.1 \mathrm{mg}$ $\left.\mathrm{mL}^{-1}\right)$ and $\mathrm{CuCl}_{2}(0.25 \mathrm{mM})$ was inoculated with $500 \mu \mathrm{L}$ of preculture. The culture was grown at $37^{\circ} \mathrm{C}(230 \mathrm{rpm})$ to reach an $\mathrm{OD}_{600}$ of $0.6 .5 \mathrm{~mL}$ of culture was centrifuged $(10 \mathrm{~min} ; 2000 \mathrm{~g} ; 4$ ${ }^{\circ} \mathrm{C}$ ) and the pellet was resuspended in $5 \mathrm{~mL}$ of fresh $\mathrm{LB}$ medium containing ampicillin $\left(0.1 \mathrm{mg} \mathrm{mL}{ }^{-1}\right), \mathrm{CuCl}_{2}(0.25 \mathrm{mM})$ and IPTG $(1 \mathrm{mM})$ for induction of protein expression. $\mathrm{OD}_{600}$ was measured and the culture was diluted to 0.2 cells per $14 \mathrm{pL}$.

The dual-dropmaker module (Fig. S2, ESI †) was used to produce simultaneously two emulsions. E. coli CotA cell suspension was encapsulated in $14 \mathrm{pL}$ droplets containing $10 \mu \mathrm{M}$ of dextran fluorescein while the library cell suspension was encapsulated in $14 \mathrm{pL}$ droplets without any marker. The binary emulsion was incubated for $15 \mathrm{~h}$ at $30^{\circ} \mathrm{C}$ and reloaded into the picoinjection module (Fig. S3, ESI $\dagger$ ). LB medium containing Amplex®UltraRed (0.5 $\mu \mathrm{M}$; Life Technologies) was used as the picoinjected phase. After $10 \mathrm{~min}$ of incubation at room temperature $\left(25^{\circ} \mathrm{C}\right)$, the emulsion was then reloaded into the reloading module (Fig. S5, ESI $\dagger$ ) for analysis.

\section{Results and discussion}

\subsection{Screening mode}

3.1.1 CotA laccase model. CotA laccase, a $65 \mathrm{kDa}$ protein, is an abundant component of the outer coat layer of endospores produced by the Gram-positive soil bacterium B. subtilis. ${ }^{29}$ It shows a particular thermostability with maximal activity at an optimal temperature of $75{ }^{\circ} \mathrm{C} .{ }^{30}$ Due to this thermal resistance, CotA is an interesting catalyst for $\mathrm{O}_{2}$-based biofuel cells (BFC), devices that use enzymatic catalysts to convert chemical energy into electrical energy. ${ }^{31}$ BFCs are especially limited by their lifetime and the catalytic activity of biocatalysts. A way to increase BFC efficiency is to use protein engineering to improve biocatalysts. ${ }^{32}$ Ideally, a BFC should work at physiological $\mathrm{pH}$ and temperature within a single compartment, which can be possible because of the high specificity of cathodic and anodic biocatalysts. The inherent thermostability of CotA laccase makes it attractive as a cathodic catalyst. It has already been shown in previous studies that CotA can be used in a BFC. ${ }^{33,34}$ However, its catalytic activity at $\mathrm{pH} 7.0$ is reduced by $80 \%$ compared to that at $\mathrm{pH} 4 .^{35}$ It would be very interesting to evolve CotA laccase for BFC applications by improving its catalytic activity under physiological $\mathrm{pH}$ conditions while preserving its thermostability.

This work reports a microfluidic screening platform to evolve recombinant CotA laccase expressed in E. coli. We used the following fluorescent assay based on the laccase enzymatic activity: CotA laccase converted the fluorogenic substrate Amplex®UltraRed (AUR) into a fluorescent dye analogous to resorufin ( $\lambda_{\max }$ excitation: $568 \mathrm{~nm} / \lambda_{\max }$ emission: $581 \mathrm{~nm}$ ). In order to validate the droplet-based microfluidic screening platform, we have constructed a frameshifted inactive variant $\Delta$ CotA.

3.1.2 $\operatorname{Cot} \mathbf{A} / \Delta \operatorname{Cot} \mathbf{A}$ activity in droplets. The activity of both E. coli CotA and E. coli $\triangle$ CotA strains was studied within droplets. Each strain was encapsulated in $14 \mathrm{pL}$ droplets containing $\mathrm{LB}$ inductive medium ( $\mathrm{LB}_{\text {ind }}$; $\mathrm{LB}$ medium $+0.1 \mathrm{mg} \mathrm{mL}^{-1}$ ampicillin $+1 \mathrm{mM}$ IPTG $+0.25 \mathrm{mM} \mathrm{CuCl}_{2}$ ). The distribution of cells between droplets during encapsulation followed the Poisson distribution. ${ }^{17}$ Cell suspensions were diluted to 0.1 cells per $14 \mathrm{pL}$ to minimize co-encapsulation events. Under these conditions, the emulsions were composed of $91 \%$ of empty droplets, $9 \%$ of droplets contained single cell and less than $1 \%$ of droplets showed double or multiple encapsulation events. This distribution was confirmed experimentally by encapsulating $E$. coli cells expressing the green fluorescent protein (data not shown). The encapsulation rate was also controlled for each experiment by imaging the emulsion after cell growth and counting droplets containing bacterial colonies.

E. coli CotA and E. coli $\triangle$ CotA strains were simultaneously encapsulated using a dual-dropmaker module and a green fluorescent barcode to tag the two populations (Fig. 2a, inset). The binary emulsion was incubated for $15 \mathrm{~h}$ at $30^{\circ} \mathrm{C}$ and then reloaded into the picoinjection device for fluorogenic substrate injection. The fluorescent signal was measured before injection (Fig. 2a) and after injection and incubation for $10 \mathrm{~min}$ at $25{ }^{\circ} \mathrm{C}$ by reloading the emulsion in a reloading module (Fig. 2b). E. coli CotA strain showed laccase activity with apparition of orange fluorescent active droplet population (Fig. 2b, 3 [0.6\%]; theoretically $1 \%$ ) while the fluorescent signal of E. coli $\triangle$ CotA droplets remained unchanged. It can also be noticed that the droplets are efficiently stabilized by surfactant, as only $0.6 \%$ of emulsion is coalesced (Fig. 2b, population 4). This is mainly due to the fact that our dropmaker devices ensured sufficient stabilization time after droplet formation and before the droplets first come into contact. ${ }^{36}$

The evolution of the fluorescent signal of the two populations over incubation time was evaluated by reloading the emulsion after $0,5,40$ and 90 min incubation after picoinjection (Fig. 2c). E. coli CotA active droplets showed a significant increase of the fluorescent signal over time while $E$. coli $\triangle$ CotA droplets showed no activity with a slight increase of the fluorescent signal. This slight increase of the fluorescent signal 

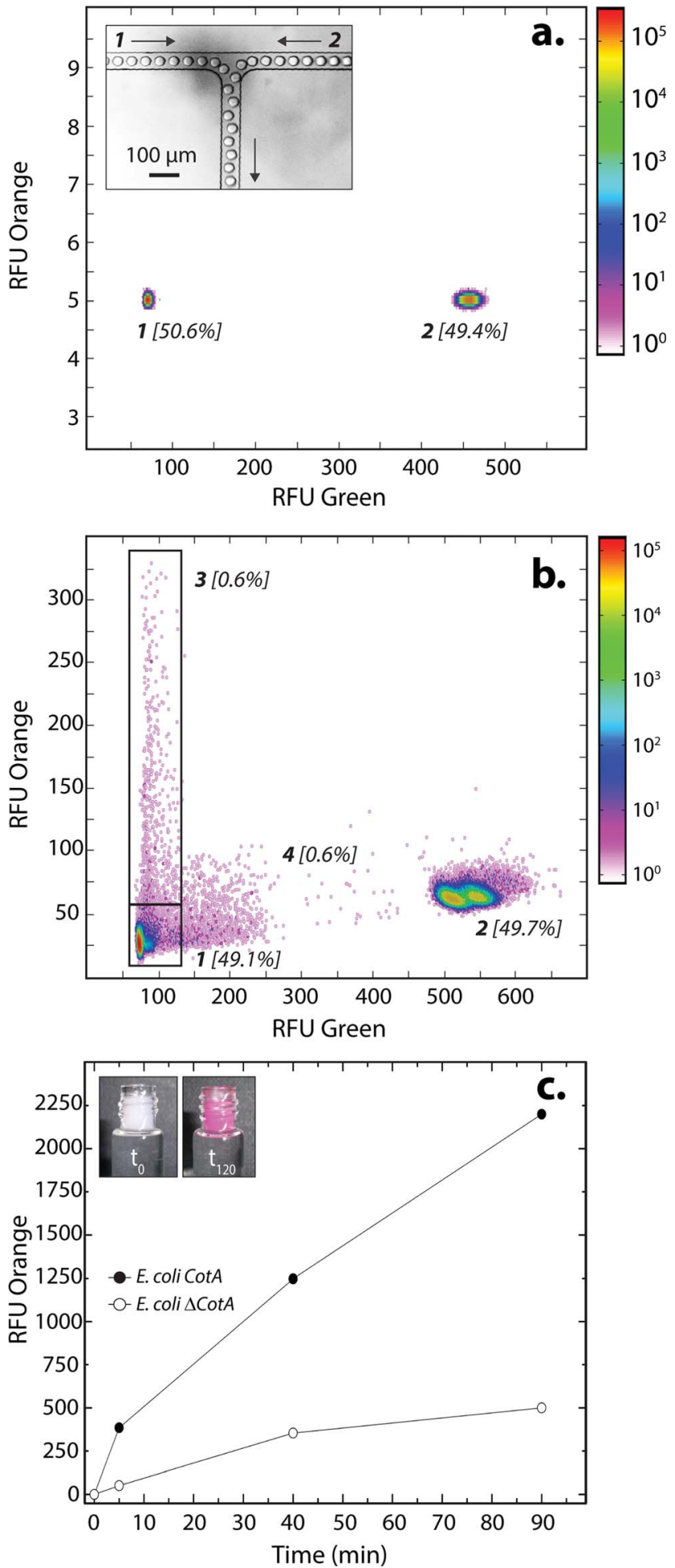

Fig. 2 E. coli $\operatorname{CotA}$ vs. E. coli $\triangle \operatorname{CotA}$ laccase activity in droplets. (a) 2D contour plot representing the green fluorescence (corresponding to barcode) and the orange fluorescence (corresponding to the $\operatorname{Cot} A$ laccase activity) of two populations of $14 \mathrm{pL}$ droplets containing LB inductive medium (LB medium + $0.1 \mathrm{mg} \mathrm{mL}^{-1}$ ampicillin + $1 \mathrm{mM} \mathrm{IPTG}$ $+0.25 \mathrm{mM} \mathrm{CuCl}_{2}$ ) after incubation for $15 \mathrm{~h}$ at $30{ }^{\circ} \mathrm{C}$. Population 1 contained $E$. coli $\operatorname{Cot} A$ and $1 \mu \mathrm{M}$ of dextran-fluorescein and population 2 contained $E$. coli $\triangle \operatorname{Cot} A$ and $5 \mu \mathrm{M}$ of dextran-fluorescein. The inset shows an image of the dual-dropmaker during encapsulation, focalizing on the area where the two emulsions combined. (b) 2D contour plot representing the green fluorescence and the orange fluorescence from the inactive $\Delta$ CotA droplets would be due to the leakage of the enzymatic reaction product, a resorufin analogue, from the active droplets mediated by the surfactant. ${ }^{37}$ The activity ratio $E$. Coli CotA : E. Coli $\triangle$ CotA increased to reach 8 at $10 \mathrm{~min}$ and then decreased to remain constant $(\sim 3)$ while equilibrium between enzymatic kinetics and leakage kinetics would be reached. According to those results, the model selection was validated using an incubation time of $10 \mathrm{~min}$ prior to sorting.

3.1.3 Model selection. The droplet-based microfluidic screening platform we developed is described in Fig. 3. Mixtures of $E$. coli $\operatorname{CotA}-E$. coli $\triangle \operatorname{CotA}$ strain cell suspensions were encapsulated in $14 \mathrm{pL}$ droplets containing inductive medium $\mathrm{LB}_{\text {ind }}$ using the dropmaker device (Fig. 3a). The emulsions were incubated at $30{ }^{\circ} \mathrm{C}$ for $15 \mathrm{~h}$ to allow cell growth and protein expression within droplets.

Droplets were reloaded in the picoinjection module (Fig. 3b) where the AUR fluorogenic substrate was picoinjected in every droplet $(\sim 1 \mathrm{pL})$. After $10 \mathrm{~min}$ of incubation at $25^{\circ} \mathrm{C}$, the droplets were reloaded in a FADS module for sorting based on the enzymatic activity according to the fluorescence intensity (Fig. 3c). The active droplet population, defined with a fluorescence sorting threshold, was sorted at a rate of $400 \mathrm{~Hz}$ and recovered. The sorted cells were grown and characterized to determine sorting efficiencies (Fig. 3d). Two colorimetric assays were developed to identify the sorted cells. In both cases, cells were first grown in LB medium. Then, cells were either recultivated separately in 96-deep well microtitre plates where the colorimetric assay was then performed (Fig. $3 \mathrm{~d}_{1}$ ) or directly plated on colorimetric inductive agar medium (Fig. $3 \mathrm{~d}_{2}$ ). The second identification method was more suitable for analyzing a large number of colonies than the first one, which was limited to 87 colonies and 9 controls per microtitre plate.

In a previous study, the fluorescence-activated droplet sorting module (FADS) has been reported to perform with a low error rate within a specific operating range. ${ }^{17}$ In that case, the main factor limiting the sorting enrichment is the co-encapsulation events of $E$. coli $\triangle$ CotA cells with $E$. coli CotA cells. A model for theoretical enrichment was previously described based on the Poisson distribution during encapsulation. ${ }^{17}$ The theoretical enrichment $\left(\eta_{\mathrm{t}}\right)$ is then given by the equation:

$$
\eta_{\mathrm{t}}=\frac{1}{1-\exp \left(-\frac{\varepsilon_{0} \lambda}{1+\varepsilon_{0}}\right)}
$$

where $\varepsilon_{0}$ is the initial ratio of $E$. coli CotA to E. coli $\Delta \operatorname{Cot} A$ and $\lambda$ is the initial mean number of cells per droplet. The enrichment is defined as the ratio of $\varepsilon$ after sorting $\left(\varepsilon_{1}\right)$ to $\varepsilon$ before sorting $\left(\varepsilon_{0}\right)$. $\lambda$ was determined for each emulsion by imaging the emulsion and using the equation (in the limit of Poisson encapsulation):

of the droplets after picoinjection of the AUR substrate and 10 min of incubation at $25^{\circ} \mathrm{C}$. Two new populations were detected: population 3 showing CotA laccase activity, and a small population 4 due to droplet coalescence. (c) Evolution of the average fluorescent signal of $E$. coli $\operatorname{Cot} A$ (dark) and $E$. coli $\triangle \operatorname{Cot} A$ (white) droplets as a function of incubation time after picoinjection. The inset shows the emulsion in the collection vial after picoinjection of the AUR substrate and $0\left(t_{0}\right)$ or 120 $\min \left(t_{120}\right)$ of incubation at $25^{\circ} \mathrm{C}$. 
a.

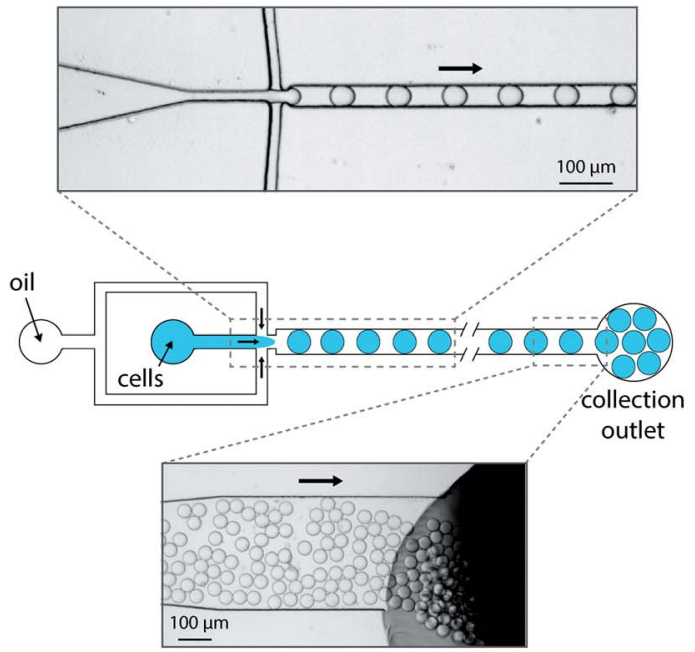

c.
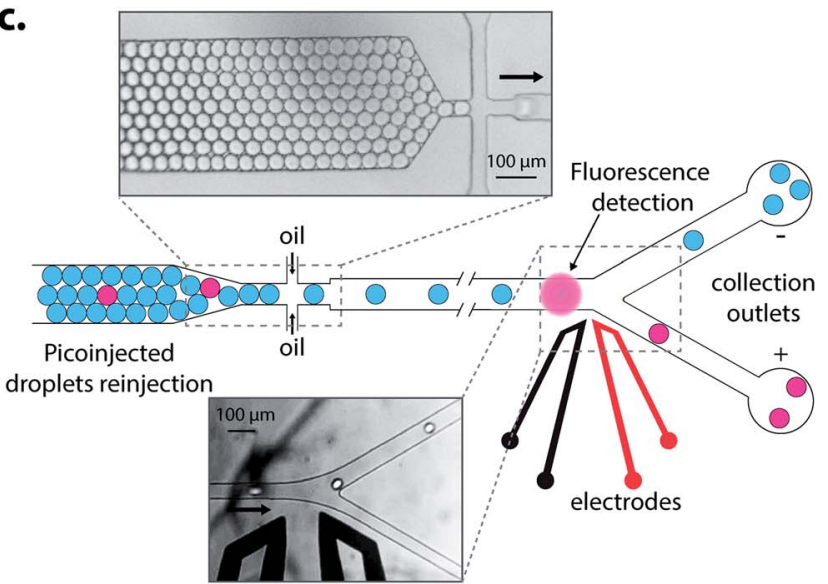

b.

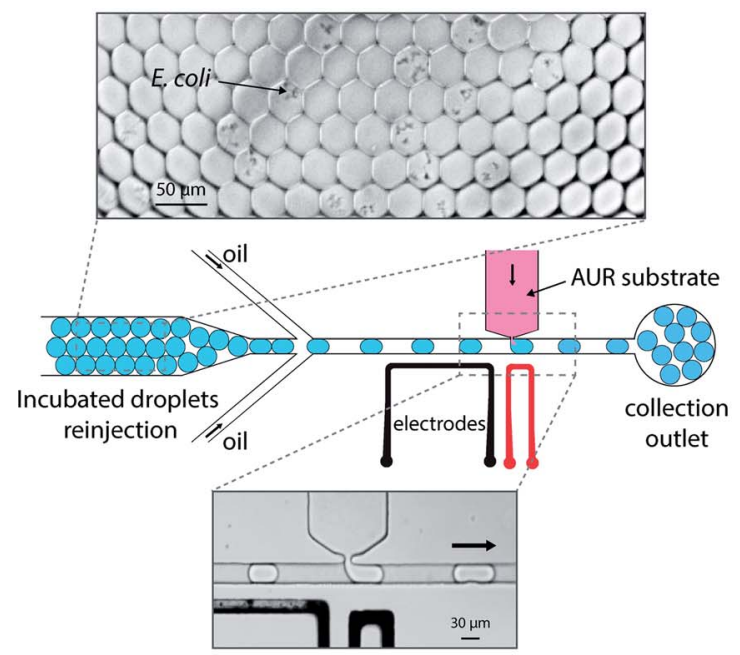

d.

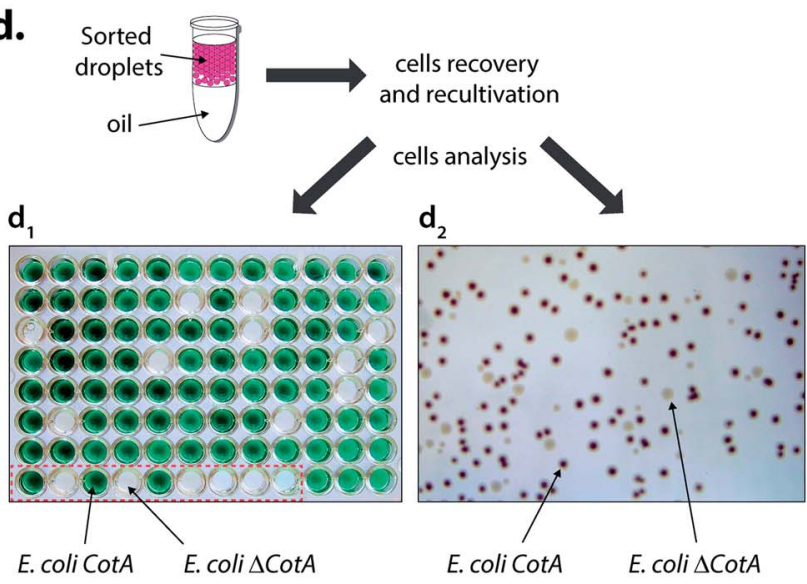

Fig. 3 Description of the droplet-based microfluidic screening platform. (a) Cell encapsulation: a mixture of E. coli CotA-E. coli $\triangle$ CotA cells in $\mathrm{LB}_{\text {ind }}$ medium was compartmentalized in $14 \mathrm{pL}$ droplets at 0.1 cell per droplet using HFE-7500 fluorinated oil containing $1 \%$ (w/w) KryJeffa900 surfactant. Once collected, the emulsion was incubated for $15 \mathrm{~h}$ at $30^{\circ} \mathrm{C}$ for cell growth and protein expression. (b) Picoinjection of the substrate: incubated droplets were reloaded and spaced using HFE-7500 fluorinated oil containing 1\% (w/w) KryJeffa900 surfactant. AUR substrate in LB medium was injected each time a droplet passed in front of the picoinjection head by application of an AC field (20 kHz, $200 \mathrm{~V})$. The emulsion was collected in ice. (c) Fluorescence-activated droplet sorting: after $10 \mathrm{~min}$ of incubation at $25^{\circ} \mathrm{C}$, the picoinjected droplets were reloaded and spaced using HFE-7500 fluorinated oil. Fluorescent-activated AC field pulses ( $30 \mathrm{kHz}, 1400 \mathrm{~V}, 0.5 \mathrm{~ms}$ ) were used to deflect fluorescent droplets in the positive thinner collection outlet. (d) Cell recovery and characterization: sorted droplets were placed in $10 \mathrm{~mL}$ of LB medium containing $0.1 \mathrm{mg} \mathrm{mL}^{-1}$ of ampicillin and incubated for $15 \mathrm{~h}$ at $37^{\circ} \mathrm{C}$. Two techniques were then used: (d1) cells were plated on LB agar medium plate. After growth, colonies were then cultivated separately in a microtitre plate. Protein expression was induced and the ABTS substrate was added. Wells showing CotA laccase activity became green while others stayed colourless. Wells in the red square are controls with $3 \mathrm{E}$. coli CotA (green) and $3 E$. coli $\triangle \operatorname{CotA}$ (colourless) and then 3 without inoculation (colourless), and (d2) cells were directly plated on a LB agar medium plate containing IPTG and ABTS substrates. After growth and coloration, colonies showing CotA laccase activity became dark while others stayed colourless.

$$
\lambda=-\ln (1-k)
$$

where $k \leq 1$ is the fraction of droplets occupied by colonies. A series of sorting was performed at a rate of $400 \mathrm{~Hz}$ using different $\varepsilon_{0}$ and $\lambda$ values to demonstrate that our screening platform followed this model. The results are shown in the table of Fig. 4. For each emulsion, the CotA active droplet population, defined by the red box sorting threshold, was sorted (Fig. $4 \mathrm{a}_{1}$ ). This sorting threshold was defined with reference to the average fluorescent signal of the inactive droplet population: in the case of a sorting threshold of 8 , droplets showing a fluorescence signal 8 times higher than the average fluorescent signal of inactive droplet population were sorted. $\varepsilon$ before sorting $\left(\varepsilon_{0}\right)$ and after sorting $\left(\varepsilon_{1}\right)$ were determined using the colorimetric assays we developed based on the CotA laccase activity (Fig. 4b). Experimental enrichments $\eta_{\exp }$ were calculated and plotted against the theoretical model (Fig. $4 \mathrm{a}_{2}$ ).

As expected, experimental data were closely fitting the theoretical model: $\eta_{\exp }$ was observed to increase decreasing $\varepsilon$ or decreasing $\lambda$. Sorting at very low $\varepsilon(0.009)$ leads to high 
a.

\begin{tabular}{llcccccc}
\hline$\lambda$ & $\varepsilon_{0}$ & \multicolumn{2}{c}{ number of droplets } & $\begin{array}{c}\text { Sorting } \\
\text { threshold }\end{array}$ & $\varepsilon_{1}$ & $\eta_{\mathrm{t}}$ & $\eta_{\exp }$ \\
\hline 0.17 & 0.16 & $1.10^{5}$ & 1500 & 8 & 7.68 & 43 & 48 \\
0.135 & 0.009 & $1.10^{5}$ & 150 & 8 & 3.93 & 831 & 437 \\
$\mathbf{0 . 0 7 1}$ & $\mathbf{0 . 0 8}$ & $\mathbf{1 . 1 0 ^ { 5 }}$ & $\mathbf{1 5 0}$ & $\mathbf{8}$ & $\mathbf{1 5 . 2 8}$ & $\mathbf{1 9 1}$ & $\mathbf{1 9 1}$ \\
0.1 & 0.9 & $1.10^{5}$ & 50 & 2 & 1.12 & 2.65 & 1.24 \\
\hline
\end{tabular}

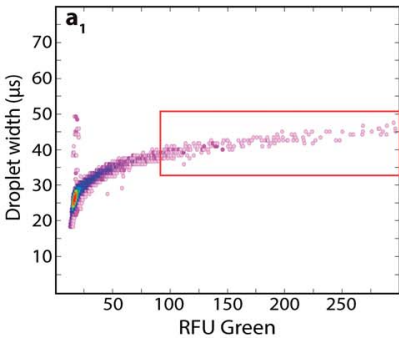

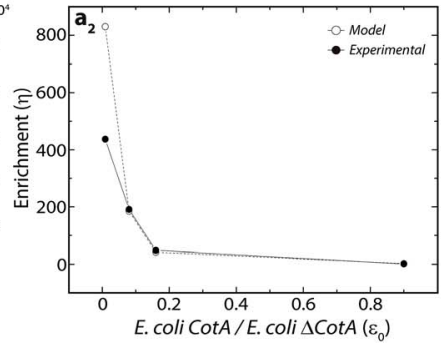

b.

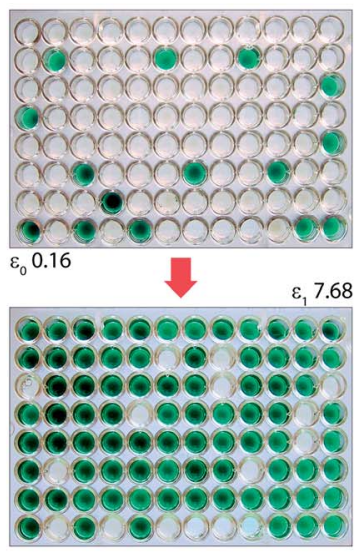

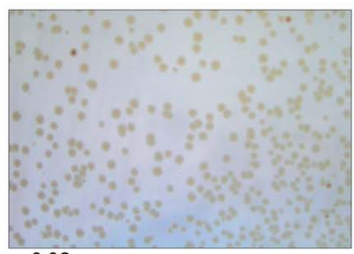
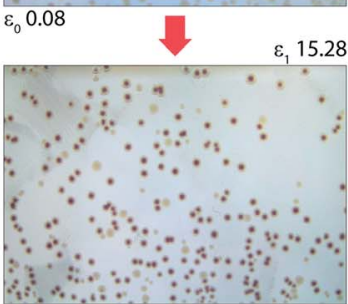

Fig. 4 Model selection of the CotA laccase activity. (a) Table summarizing the results for different sorts: $\lambda$, $\varepsilon_{0}, \varepsilon_{1}$, theoretical ( $\eta_{t}$ ) and experimental $\left(\eta_{\text {exp }}\right)$ enrichments. $\left(a_{1}\right)$ 2D contour plot representing the width of the droplets (transit time of the droplet passing the detector; $\mu$ s) and the orange fluorescence (corresponding to the CotA laccase activity) of $14 \mathrm{pL} \mathrm{LB}$ ind droplets after compartmentalization of a mixture of $E$. coli CotA/E. coli $\triangle \mathrm{Cot} A$, incubation for $15 \mathrm{~h}$ at $30^{\circ} \mathrm{C}$, picoinjection of the AUR fluorogenic substrate and incubation for $10 \mathrm{~min}$ at $25^{\circ} \mathrm{C}$. Population defined in the red box sorting threshold was sorted. Cell mixtures before and after sorting were analyzed to compare the initial $\left(\varepsilon_{0}\right)$ and final $\left(\varepsilon_{1}\right)$ ratios of $E$. coli $\operatorname{Cot} A$ to $E$. coli $\triangle \operatorname{Cot} A$ cells. $\left(\mathrm{a}_{2}\right)$ Evolution of sorting enrichment as a function of $\varepsilon_{0}$ for the theoretical model (white dots) and experimental data (black dots). (b) Picture of the colorimetric assays performed to determine $\varepsilon$ ratios before $\left(\varepsilon_{0}\right)$ and after sorting $\left(\varepsilon_{1}\right)$. Microtitre plates on the left refer to data in italic in the table while Petri dishes on the right refer to data in bold in the table.

enrichment. $\eta_{\exp }$ is only half $\eta_{\mathrm{t}}$ in that case, which can be explained by the lower sorting threshold (2) implying that more false positive droplets were caught. Those results highlighted the fact that this screening technique offers two operation modes. Working at high $\lambda$ implies more co-encapsulation events ( $26 \%$ for $\lambda=1$ ) but higher throughput ( 250 cells per $\mathrm{s}$ for $\lambda=1$ ) and is suitable for fast enrichment of a library before further fine selection steps. Working with low $\lambda(\lambda<0.2)$ implies less coencapsulation events $(1.8 \%)$ and lower throughput (70 cells per s) and is suitable for sorting cells to high purities for fine selection steps.

The major advantage of this droplet-based microfluidic platform relies on the flexibility brought by the injection of the substrate at a defined time. This allows us to adapt the incubation time prior to sorting to the kinetics of the enzymatic reaction and therefore makes the selection occur in the linear range of the kinetics.

\subsection{Analysis mode}

The droplet-based platform can also operate in analysis mode in which the droplet fluorescence is measured in a reloading module without any sorting. In that case, as FADS is the limiting module in terms of throughput, the analysis of droplets can be performed at very high rates up to $2000 \mathrm{~Hz}$. We constructed a library of $10^{6}$ variants using error-prone PCR. Amplified fragment was purified using biotin-streptavidin binding and magnetic beads and then cloned into an acceptor vector constructed from pET-21a $(+)$. The library characteristics were evaluated by sequencing twenty variants. It showed, on average, 910 mutations per gene that is roughly $2-4$ amino acid mutations per variant.

The enzymatic activity distribution of this library was analyzed using CotA wild type as an internal reference: $E$. coli
CotA and the library cell suspensions were separately and simultaneously encapsulated $(\lambda=0.2)$ using the dual-dropmaker module. The binary emulsion was incubated for $15 \mathrm{~h}$ at $30{ }^{\circ} \mathrm{C}$ and then reloaded into the picoinjection device for fluorogenic substrate injection. After $10 \mathrm{~min}$ of incubation at $25^{\circ} \mathrm{C}$, the enzymatic activity was measured at 1000 droplets per $\mathrm{s}$ in a reloading module (Fig. 5). A green fluorescent barcode was used to tag the two populations: the CotA internal reference (1 [52\%])

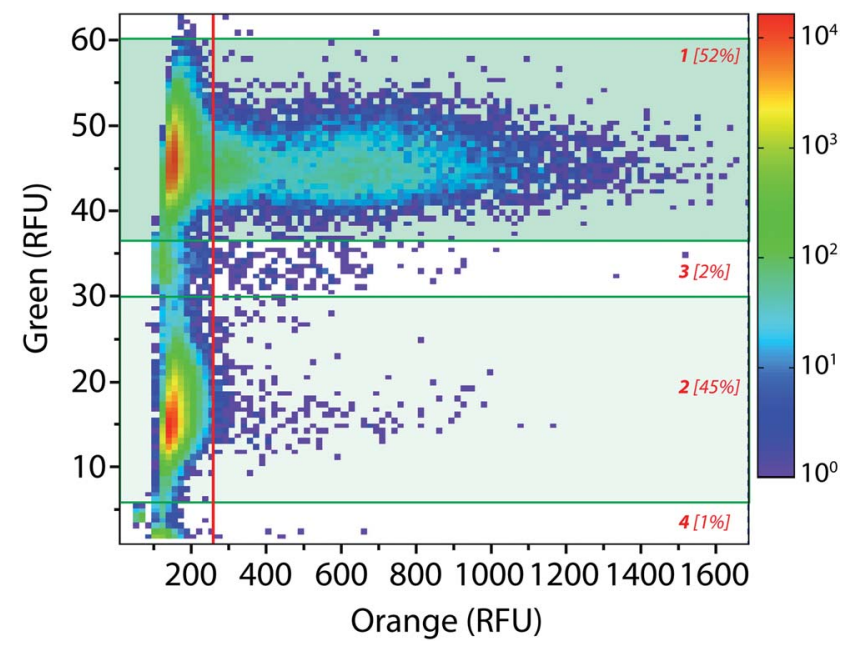

Fig. 5 High-throughput library analysis: 2D contour plot representing the green fluorescence (barcode) and the orange fluorescence (enzymatic activity) of a wild type vs. library binary emulsion after picoinjection of the AUR substrate and $10 \mathrm{~min}$ of incubation at $25^{\circ} \mathrm{C}$. Population [1] refers to wild type CotA internal reference droplets (10 $\mu \mathrm{M}$ dextran-fluorescein barcode) and population [2] refers to the library droplets (2 $\mu \mathrm{M}$ dextran-fluorescein barcode). Populations [3] and [4] correspond respectively to coalesced droplets and unpicoinjected droplets. 
and the library $(2[45 \%])$. The encapsulation rate was measured to be $15 \%$ of occupied droplets and $85 \%$ of empty one. For each of the sub-population, the active population was defined using a 250 RFU threshold (red line in Fig. 5): the wild type reference with an average fluorescence signal of 625 RFU (11\%) and the library with an average fluorescence signal of 425 RFU (0.25\%). This high-throughput analysis (75 variants per s) showed that the library contained approximately $2.5 \%$ of active mutants or unmutated CotA. As the mutation rate is rather high in this library, it is worth considering that the proportion of unmodified wild type is quite low. Those $2.5 \%$ active variants were on average 1.5 times less active than the wild type CotA. This activity distribution within the library is not surprising as the mutation rate is rather high. This platform allows analysis of libraries using high throughputs. In this example, 150 occupied droplets are analyzed in one second, half of them referred to the wild type and the other half to the library. In that case, 75 variants are analyzed per second, $10^{6}$ in less than 4 hours. It is possible to work at higher $\lambda$ values that will increase the throughput: $\lambda=0.5$ allows analysis of $10^{6}$ cells in 1.5 hours with only $9 \%$ of co-encapsulation events. Moreover, the internal wild type reference could be used in a smaller ratio than $1: 1$ with the library, which would increase the throughput of variant analysis. It is then also possible to increase the analysis rate up to $2000 \mathrm{~Hz}$. In that case, with an internal reference-library $1: 9$ ratio, $10^{6}$ cells are analyzed in less than $1 \mathrm{~h}(\lambda=0.2)$ or in $20 \mathrm{~min}$ $(\lambda=0.5)$. Additional gain in analytical throughput might be obtained by parallelization. ${ }^{38}$ The efficiency and the throughput could also be theoretically improved by organizing the cells during encapsulation to avoid the Poisson distribution and screen the fully loaded emulsion with single cells per droplet. ${ }^{39}$

This high-throughput analysis droplet-based microfluidic platform offers a precious tool for directed evolution experiments based on the enzymatic activity. It allows the possibility to rapidly study the activity distribution of large libraries with a tremendous saving of time and reagents. Using this new technology, it is possible to follow the distribution of the activity after each round of mutagenesis or selection. That could be very helpful for choosing mutagenesis methods and directed evolution strategies to follow a desired evolution path and avoid dead ends.

\section{Conclusions}

We have demonstrated a flexible and efficient droplet-based screening technique able to analyze and sort cells based on a recombinant enzymatic activity. The main merit of this screening platform is the injection of the fluorogenic substrate at a defined time, which offers the possibility to tune the incubation time prior to selection depending on the kinetics of the enzymatic reaction. This high-throughput droplet-based microfluidic platform for directed evolution experiments is used to: (i) evaluate the distribution of the enzymatic activity within a large library at high-throughput (analysis mode; up to $10^{6}$ variants in $1 \mathrm{~h}$ ), (ii) rapidly enrich a library in active variants in a high-throughput rate (enrichment mode; up to $10^{6}$ variants in $1 \mathrm{~h}$ ), and (iii) select finely variants depending on their enzymatic activity (selection mode; up to $10^{6}$ variants in $4 \mathrm{~h}$ ).

The screening platform is flexible and could be applied to many kinds of recombinant systems implying ideally the secretion of the enzyme or the display of the enzyme at the cell surface, but also, as in the case of CotA laccase, periplasmic or cytoplasmic enzymes (the lysis of part of the cells within droplets would be required for the substrate to access the enzyme). Moreover, the incubation time prior to sorting could be adapted according to the enzyme kinetics. This platform also gives access to a wide range of controlled selection pressures $(\mathrm{pH}$, temperature, ...) as the growth of cells and the enzymatic assay are two distinct steps.

It is worth noting that incubating droplets off-chip for such an enzymatic reaction time scale (0-60 min) introduces a small heterogeneity within the incubation time of each droplet. We are developing an integrated device that can perform picoinjection, incubation on-chip and sorting to attempt higher degrees of control of the time and temperature of incubation. For the longer enzymatic reaction time scale (>60 $\mathrm{min}$ ), incubation off-chip is necessary and implies that the product of the enzymatic reaction is not leaking out of droplets (which could be overcome by chemical modification of the substrate to make the product more hydrophilic ${ }^{40}$ ). Such developments should lead to very robust and universal high-throughput screening techniques for directed evolution.

\section{Acknowledgements}

T.B. thanks the ADEME (Agence de Développement de l'Environnement et la Maîtrise de l'Energie) and the Region Alsace for financial support. This work was also supported by the Ministère de l'Enseignement Supérieur et de la Recherche and the Centre National de la Recherche Scientifique (CNRS). F.C. was supported by FP-7 FET OPEN Project E-Flux. The authors would like to thank Jean-François Bartolo for surfactant synthesis, Chaouki Ben Salem for technical assistance and Antoine Drevelle for useful scientific discussions.

\section{Notes and references}

1 S. Bershtein and D. S. Tawfik, Curr. Opin. Chem. Biol., 2008, 12, 151.

2 J. D. Keasling, ACS Chem. Biol., 2008, 3, 64.

3 S. G. Peisajovich and D. S. Tawfik, Nat. Methods, 2007, 4, 991.

4 L. M. Mayr and P. Fuerst, J. Biomol. Screening, 2008, 13, 443.

5 T. Thorsen, S. J. Maerkl and S. R. Quake, Science, 2002, 298, 580.

6 J. Lay, R. Liyanage, S. Borgmann and C. L. Wilkins, TrAC, Trends Anal. Chem., 2006, 25, 1046.

7 D. S. Tawfik and A. D. Griffiths, Nat. Biotechnol., 1998, 16, 652.

8 A. D. Griffiths and D. S. Tawfik, Trends Biotechnol., 2006, 24, 395.

9 R. Seemann, M. Brinkmann, T. Pfohl and S. Herminghaus, Rep. Prog. Phys., 2012, 75, 016601. 
10 L. Mazutis, J.-C. Baret and A. D. Griffiths, Lab Chip, 2009, 9, 2665.

11 K. Ahn, J. Agresti, H. Chong, M. Marquez and D. Weitz, Appl. Phys. Lett., 2006, 88, 264105.

12 L. Frenz, A. El Harrak, M. Pauly, S. Bégin-Colin, A. D. Griffiths and J.-C. Baret, Angew. Chem., Int. Ed., 2008, 47, 6817.

13 A. R. Abate, T. Hung, P. Mary, J. J. Agresti and D. A. Weitz, Proc. Natl. Acad. Sci. U. S. A., 2010, 107, 19163.

14 S. L. Sjostrom, H. N. Joensson and H. Andersson Svahn, Lab Chip, 2013, 13, 1754.

15 L. Frenz, K. Blank, E. Brouzes and A. D. Griffiths, Lab Chip, 2009, 9, 1344.

16 D. Pekin, Y. Skhiri, J.-C. Baret, D. Le Corre, L. Mazutis, C. Ben Salem, F. Millot, A. El Harrak, J. B. Hutchison, J. W. Larson, D. R. Link, P. Laurent-Puig, A. D. Griffiths and V. Taly, Lab Chip, 2011, 11, 2156.

17 J.-C. Baret, O. J. Miller, V. Taly, M. Ryckelynck, A. El Harrak, L. Frenz, C. Rick, M. L. Samuels, J. B. Hutchison, J. J. Agresti, D. R. Link, D. A. Weitz and A. D. Griffiths, Lab Chip, 2009, 9, 1850.

18 M. T. Guo, A. Rotem, J. A. Heyman and D. A. Weitz, Lab Chip, 2012, 12, 2146.

19 A. Fallah-Araghi, J.-C. Baret, M. Ryckelynck and A. D. Griffiths, Lab Chip, 2012, 12, 882.

20 S. L. Sjostrom, Y. Bai, M. Huang, Z. Liu, J. Nielsen, H. N. Joensson and H. Andersson Svahn, Lab Chip, 2014, 14, 806.

21 J. J. Agresti, E. Antipov, A. R. Abate, K. Ahn, A. C. Rowat, J.-C. Baret, M. Marquez, A. M. Klibanov, A. D. Griffiths and D. A. Weitz, Proc. Natl. Acad. Sci. U. S. A., 2010, 107, 4004.

22 B. Kintses, C. Hein, M. F. Mohamed, M. Fischlechner, F. Courtois, C. Lainé and F. Hollfelder, Chem. Biol., 2012, 19, 1001.

23 F. J. Enguita, L. O. Martins, A. O. Henriques and M. A. Carrondo, J. Biol. Chem., 2003, 278, 19416.
24 M. Fernández-Fernández, M. Ángeles Sanromán and D. Moldes, Biotechnol. Adv., 2013, 31, 1808.

25 D. C. Duffy, J. C. McDonald, O. J. A. Schueller and G. M. Whitesides, Anal. Chem., 1998, 70, 4974.

26 A. C. Siegel, D. A. Bruzewicz, D. B. Weibel and G. M. Whitesides, Adv. Mater., 2007, 19, 727.

27 C. Holtze, A. C. Rowat, J. J. Agresti, J. B. Hutchison, F. E. Angile, C. H. J. Schmitz, S. Koester, H. Duan, K. J. Humphry, R. A. Scanga, J. S. Johnson, D. Pisignano and D. A. Weitz, Lab Chip, 2008, 8, 1632.

28 B. Miroux and J. E. Walker, J. Mol. Biol., 1996, 260, 289.

29 M. F. Hullo, I. Moszer, A. Danchin and I. Martin-Verstraete, J. Bacteriol., 2001, 183, 5426.

30 L. O. Martins, C. M. Soares, M. M. Pereira, M. Teixera, T. Costa, G. H. Jones and A. O. Henriques, J. Biol. Chem., 2002, 277, 18859.

31 A. Zebda, C. Gondran, A. Le Goff, M. Holzinger, P. Cinquin and S. Cosnier, Nat. Commun., 2011, 2, 370.

32 F. Durand, C. Stimes-Chaumiel, V. Flexer, I. André and N. Mano, Biochem. Biophys. Res. Commun., 2010, 402, 750.

33 T. Beneyton, A. El Harrak, A. D. Griffiths, P. Hellwig and V. Taly, Electrochem. Commun., 2011, 13, 24.

34 T. Beneyton, I. Putu Mahendra Wijaya, C. Ben Salem, A. D. Griffiths and V. Taly, Chem. Commun., 2013, 49, 1094.

35 P. Durao, Z. Chen, C. S. Silva, C. M. Soares, M. M. Pereira, S. Todorovic, P. Hildebrandt, I. Bento, P. F. Lindley and L. O. Martins, Biochem. J., 2008, 412, 339.

36 J.-C. Baret, F. Kleinschmidt, A. El Harrak and A. D. Griffiths, Langmuir, 2009, 25, 6088.

37 T. Chen, A. Wijaya Gani and S. K. Y. Tang, Lab Chip, 2012, 12, 5093.

38 J. Lim, J. Vrignon, P. Gruner, C. S. Karamitros, M. Konrad and J.-C. Baret, Appl. Phys. Lett., 2013, 203704.

39 J. F. Edd, D. Di Carlo, K. J. Humphry, S. Köster, D. Irimia, D. A. Weitz and M. Toner, Lab Chip, 2008, 8, 1262.

40 M. Najah, E. Mayot, I. Putu Mahendra Wijaya, A. D. Griffiths, S. Ladame and A. Drevelle, Anal. Chem., 2013, 85, 9807. 